\title{
WEAKLY PRIMAL SUBMODULES
}

\author{
S. EBRAHIMI ATANI AND A. YOUSEFIAN DARANI
}

\begin{abstract}
Let $R$ be a commutative ring and let $M$ be an $R$-module. A submodule $N$ of $M$ is called a weakly primal submodule provided that the set $P=w(N) \cup\{0\}$ forms an ideal of $R$. Here $w(N)$ is the set of elements of $R$ that are not weakly prime to $N$, where an element $r \in R$ is not weakly prime to $N$ if $0 \neq r m \in N$ for some $m \in M \backslash N$. In this paper we give some basic results about weakly primal submodules. Also we discuss on the relations between the classes of the weakly primal submodules of $M$ and the weakly primal submodules of modules of fractions of $M$.
\end{abstract}

\section{Introduction}

Throughout this paper all rings are commutative with a nonzero identity and all modules are unitary. The concept of primal submodules has been introduced and studied by J. Dauns in [2] (See also [5]). Let $R$ be a commutative ring and let $M$ be an $R$-module. An element $r \in R$ is said to be prime to $N$ if $r m \in N$ (with $m \in M$ ) implies that $m \in N$. Denote by $S(N)$ the set of all elements of $R$ that are not prime to $N$. $N$ is called a primal submodule of $M$ if $S(N)$ forms an ideal of $R$. In this case $P=S(N)$ is a prime ideal of $R$, called the adjoint ideal of $N$. We also say that $N$ is a $P$-primal submodule of $M$. In this paper we give a new class of submodules: Weakly primal submodules.

First let us to introduce some definitions and notions we will use throughout. Let $R$ be a commutative ring. A proper ideal $P$ of $R$ is said to be weakly prime if $0 \neq a b \in P$ implies that $a \in P$ or $b \in P([1])$. Assume that $N$ is a submodule of an $R$-module $M$. Denote by $\left(N:_{R} M\right)$ the set of all elements $r \in R$ with $r M \subseteq N$. In this case $\left(0:_{R} M\right)$ is the annihilator of $M$. $M$ is called a faithful $R$-module if $\left(0:_{R} M\right)=0$. An element $r \in R$ is called a zero-divisor on $M$ provided that there exists $0 \neq m \in M$ such that $r m=0$. An element $r \in R$ which is not a zero-divisor on $M$ is called an $M$-regular element.

Here we shortly summarize the content of the paper. In Section 2, we give some basic results about weakly primal submodules. We provide a characterization of weakly primal submodules in Theorem 2.4. It is shown in Proposition 2.5 that if $N$ is a $P$-weakly primal submodule of $M$, then $P$ is a weakly prime ideal of $R$. In Example 2.9 we see that for a weakly primal submodule $N$ of $M$, the ideal $\left(N:_{R} M\right)$ need not necessarily be a weakly primal ideal of $R$. But if $M$ is a faithful cyclic $R$-module, then $\left(N:_{R} M\right)$ is a weakly

Received July 3, 2008.

2000 Mathematics Subject Classification. 13C05, 13C13.

Key words and phrases. Weakly primal, primal, weakly prime. 
primal ideal of $R$ (See Proposition 2.8). The concepts "primal submodule" and "weakly primal submodule" are different. In fact neither implies the other (See Examples 2.10 and 2.11). In Theorem 2.12 we prove that if $N$ is a $P$-weakly primal submodule of $M$ with $\left(N:_{R} M\right) \subseteq P$ and $N\left(N:_{R} M\right) \neq 0$, then $N$ is primal.

Let $R$ be a commutative ring, $S$ a multiplicatively closed subset of $R$ and $M$ an $R$-module. Consider the $S^{-1} R$-module $S^{-1} M$. In Section 3 we investigate the relations between the weakly primal submodules of $M$ and the weakly primal submodules of $S^{-1} M$. Let $N$ be a submodule of an $R$-module $M$. It is known that the equality $S^{-1}\left(N:_{R} M\right)=\left(S^{-1} N:_{S^{-1} R} S^{-1} M\right)$ does not necessarily hold. But in Proposition 3.1. we see that if $S$ consists of $M$-regular elements of $R$, and $N$ a $P$-weakly primal submodule of $M$ with $P \cap S=\emptyset$, then we have this equality. It is shown in Theorem 3.4 that there exists a one-to-one correspondence between the $P$-weakly primal submodules of $M$ and the $S^{-1} P$-weakly primal submodules of $S^{-1} M$, in which $S$ is a multiplicativley closed subset of $M$-regular elements of $R$ and $P$ a weakly prime ideal of $R$ with $P \cap S=\emptyset$.

\section{Basic results}

Definition 2.1. Let $N$ be a submodule of an $R$-module $M$. An element $r \in R$ is called weakly prime (simply $w p)$ to $N$ if $0 \neq r m \in N(m \in M)$ implies that $m \in N$. Otherwise $r$ is not weakly prime (simply $n w p$ ) to $N$. Denote by $w(N)$ the set of elements of $R$ that are $n w p$ to $N$.

Remark 2.2. Let $N$ be a submodule of an $R$-module $M$. Then:

(1) 0 is always weakly prime to $N$.

(2) If $r \in R$ is prime to $N$, then $r$ is $w p$ to $N$, but the converse is not necessarily true. For example consider the $\mathbb{Z}$-module $M=\mathbb{Z} / 24 \mathbb{Z}$ and it's submodule $N=8 \mathbb{Z} / 24 \mathbb{Z}$. Denote each coset $a+24 \mathbb{Z}$ in $M$ by $\bar{a}$. Then, as $6 \cdot \overline{12}=\overline{0} \in N, 6$ is not prime to $N$. But if $6 . \bar{a} \in N$ for some $\bar{a} \in M$, then 4 divides $a$. Hence $6 . \bar{a}=\overline{0}$. This implies that 6 is $w p$ to $N$.

Definition 2.3. Let $R$ be a commutative ring and let $N$ be a proper submodule of an $R$-module $M . N$ is called weakly primal if the set $P=w(N) \cup\{0\}$ forms an ideal of $R$. $P$ is called the (weakly) adjoint ideal of $N$ and we also say that $N$ is a $P$-weakly primal submodule of $M$.

The following Theorem provides a characterization of weakly primal submodules.

Theorem 2.4. Let $P$ be an ideal of a commutative ring $R, M$ an $R$-module and $N$ a submodule of $M$. The following statements are equivalent:

(a) $N$ is P-weakly primal.

(b) For every $r \notin P-\{0\},\left(N:_{M} r\right)=N \cup(0: M r)$; and for every $0 \neq r \in P$, $N \cup\left(0:_{M} r\right) \varsubsetneqq\left(N:_{M} r\right)$. 
Proof. $\quad($ a) $\Rightarrow(b)$ Suppose that $N$ is a $P$-weakly primal submodule of $M$. Then $w(N)=P \backslash\{0\}$. Let $r \notin P \backslash\{0\}$, and choose an element $m \in\left(N:_{M} r\right)$. If $r m=0$, then $m \in\left(0:_{M} r\right)$. If $r m \neq 0$, since $r$ weakly prime to $N$ we get $m \in N$. Hence $m \in N \cup\left(0:_{M} r\right)$, that is $\left(N:_{M} r\right) \subseteq N \cup\left(0:_{M} r\right)$. Therefore $\left(N:_{M} r\right)=N \cup\left(0:_{M} r\right)$.

Now assume that $r \in P \backslash\{0\}=w(N)$. Then $r$ is not weakly prime to $N$. So there exists $m \in M \backslash N$ such that $0 \neq r m \in N$. Hence $m \in\left(N:_{M} r\right) \backslash\left(N \cup\left(0:_{M} r\right)\right)$.

(b) $\Rightarrow($ a) It follows from (b) that $w(N)=P \backslash\{0\}$. Hence $N$ is $P$-weakly primal.

Proposition 2.5. Let $R$ be a commutative ring, and let $M$ be an $R$-module. If $N$ is a $P$-weakly primal submodule of $M$ then $P$ is a weakly prime ideal of $R$.

Proof. Suppose that $r, s \in R \backslash P$ are such that $r s \neq 0$. If there is $m \in M$ with $0 \neq(r s) m \in N$, then $0 \neq r m \in\left(N:_{M} s\right)=N \cup\left(0:_{M} s\right)$ by Theorem 2.4. Hence $0 \neq r m \in N$. As $r \notin P, r$ is weakly prime to $N$. Hence $m \in N$, that is $r s$ is weakly prime to $N$. So $r s \notin P$. Consequently $P$ is a weakly prime ideal of $R$.

Example 2.6. It is shown in [5, Proposition 2.2] that if $N$ is a $P$-primal submodule of an $R$-module $M$, then $\left(N:_{R} M\right) \subseteq P$. Let $N$ be a $P$-weakly primal submodule of $M$. Contrary to what happens in a primal submodule, the ideal $\left(N:_{R} M\right)$ is not, in general, contained in $P$. For example consider the $\mathbb{Z}$-module $M=\mathbb{Z} / 8 \mathbb{Z}$. Denote by $\bar{a}$ each coset $a+8 \mathbb{Z}$ in $M$. If we put $N=\{\overline{0}\}$, then $W(N)=\emptyset$. Thus $N$ is a 0 -weakly primal submodule of $M$. But $\left(N:_{R} M\right)=8 \mathbb{Z} \nsubseteq\{0\}$. Now consider the following result.

Proposition 2.7. Let $N$ be a faithful submodule of an $R$-module $M$. Then:

(1) If $N$ is a $P$-weakly primal submodule of $M$, then $\left(N:_{R} M\right) \subseteq P$.

(2) If $N$ is a 0 -weakly primal, then $M / N$ is a faithful $R$-module.

Proof. (1) Assume that $N$ is a $P$-weakly primal. Since $N$ is a proper submodule, $\left(N:_{R} M\right)$ is a proper ideal of $R$ and there exists $m \in M \backslash N$. Assume that $r$ is a nonzero element of $\left(N:_{R} M\right)$. Then $r m \in N$. If $r m \neq 0, r$ is nor weakly prime to $N$, that is $r \in P$. If $r m=0$, there exists $n \in N$ with $r n \neq 0$. Now $0 \neq r(m+n) \in N$ with $m+n \notin N$ implies that $r$ is not weakly prime to $N$, that is $r \in P$. Thus $\left(N:_{R} M\right) \subseteq P$.

(2) It follows from (1).

Proposition 2.8. Let $R$ be a commutative ring, $M$ a faithful cyclic $R$-module and $N$ a $P$-weakly primal submodule of $M$. Then $\left(N:_{R} M\right)$ is a $P$-weakly primal ideal of $R$.

Proof. Assume that $M=R x$ for some $x \in M$, and set $I=\left(N:_{R} M\right)$. We show that $w(I)=w(N)$. For every $r \in w(I)$, there exists $a \in R \backslash I$ with $0 \neq r a \in I$. In this case $\operatorname{rax} \neq 0$ since otherwise $r a \in\left(0:_{R} x\right)=\left(0:_{R} M\right)=0$ which is a contradiction. As $a x \in M \backslash N$ it follows that $r$ is nwp to $N$, that is $r \in w(N)$. So $w(I) \subseteq w(N)$. Now assume that $r \in w(N)$. Then $0 \neq r m \in N$ for some $m \in M \backslash N$. We can write $m=r^{\prime} x$ for some $r^{\prime} \in R$. Then $0 \neq r r^{\prime} x \in N$. This implies that $0 \neq r r^{\prime} \in I$ with $r^{\prime} \in R \backslash I$, that is $r$ is nwp to $I$. Hence $r \in w(I)$ and so $w(N) \subseteq w(I)$. 
Example 2.9. Let $R$ be a commutative ring. It is known that for every prime submodule $N$ of an $R$-module $M$, the ideal $\left(N:_{R} M\right)$ is a prime ideal of $R$. Assume that $N$ is a weakly primal submodule of $M$ which is not prime. In general, $\left(N:_{R} M\right)$ need not be weakly primal. For example consider the $\mathbb{Z}$-module $M=\mathbb{Z}_{6}$ and let $N=0$. By Example 2.11, $N$ is a weakly primal submodule of $M$. But $\left(N:_{R} M\right)=6 \mathbb{Z}$ is not a weakly primal ideal of $\mathbb{Z}$ ( since $0 \neq 2.3 \in 6 \mathbb{Z}$, that is $2,3 \in w(N)$ while $3-2=1 \notin w(N)$ ). This example shows that the condition " $\mathrm{M}$ is faithful" is not superficial in Proposition 2.8 .

The concepts "primal submodule" and "weakly primal submodule" are different. In fact, neither implies the other. We will show this by the following examples. In Example 2.10 we give a primal submodule that is not weakly primal. An example of a weakly primal submodule which is not primal is given in Example 2.11.

Example 2.10. Consider the submodule $N=8 \mathbb{Z} / 24 \mathbb{Z}$ of the $\mathbb{Z}$-module $M=\mathbb{Z} / 24 \mathbb{Z}$. Denote each coset $a+24 \mathbb{Z}$ in $M$ by $\bar{a}$.

(1) Since $0 \neq 2 . \overline{4} \in N$ and $0 \neq 4 . \overline{2} \in N$ with $\overline{2}, \overline{4} \in M \backslash N$, we have $2,4 \in w(N)$. If $6 . \bar{a} \in N$ for some $\bar{a} \in M$, then 4 divides $a$, and hence $6 . \bar{a}=0$. This shows that $2+4=6$ is $w p$ to $N$. Therefore $w(N) \cup\{0\}$ is not an ideal of $\mathbb{Z}$, that is $N$ is not a weakly primal submodule of $M$.

(2) It is clear that $M / N \cong \mathbb{Z}_{8}$ and $\mathbb{Z} /\left(N:_{\mathbb{Z}} M\right) \cong \mathbb{Z}_{8}$. Moreover $Z d v_{\mathbb{Z}_{8}}\left(\mathbb{Z}_{8}\right)$ is an ideal of $\mathbb{Z}_{8}$. This implies that $Z d v_{R /(N: \mathbb{Z} M)}(M / N)$ is an ideal of $\mathbb{Z} /\left(N:_{\mathbb{Z}} M\right)$. It follows from [5, Theorem 2.3] that $N$ is a primal submodule of $M$.

This example shows that a primal submodule need not be weakly primal.

Example 2.11. Consider the $\mathbb{Z}$-module $M=\mathbb{Z}_{6}$, and denote every integer $a$ modulo 6 by $\bar{a}$. Consider the submodule $N=\{\overline{0}\}$ of $M$. Then:

(1) Clearly $w(N)=\emptyset$. So $N$ is a weakly primal submodule of $M$.

(2) Since $2 . \overline{3}=\overline{0} \in N$ and $3 . \overline{2}=\overline{0} \in N$, we have $2,3 \in S(N)$, while $3-2=1$ is prime to $N$. Therefore $N$ is not a primal submodule of $M$.

This example shows that a weakly primal submodule need not be primal.

Now we have the following Theorem.

Theorem 2.12 Let $M$ be an $R$-module and $N$ a $P$-weakly primal submodule of $M$ with $\left(N:_{R} M\right) \subseteq P$ and $N\left(N:_{R} M\right) \neq 0$. Then $N$ is a primal submodule of $M$.

Proof. It suffices to show that $P=S(N)$. For every $0 \neq r \in P, r$ is nwp to $N$. Hence, by Remark 2.2, $r$ is not prime to $N$, that is $r \in S(N)$. So $P \subseteq S(N)$. Now assume that $a \in S(N)$. There exists $m \in M \backslash N$ with $a m \in N$. If $a m \neq 0$, then $a$ is $n w p$ to $N$ and so $a \in P$. So assume that $a m=0$. Consider the two following cases:

Case 1. $a N \neq 0$. There exists $n \in N$ with $a n \neq 0$. Now $0 \neq a(m+n) \in N$ with $m+n \in M \backslash N$ implies that $a$ is $n w p$ to $N$, and hence $a \in P$. 
case 2. $a N=0$. If $m\left(N:_{R} M\right) \neq 0$, then $r m \neq 0$ for some $r \in\left(N:_{R} M\right)$. Now $0 \neq(a+r) m \in N$ with $m \in M \backslash N$ implies that $a+r$ is $n w p$ to $N$, that is $a+r \in P$ and hence $a \in P$. If $m\left(N:_{R} M\right)=0$, since $N\left(N:_{R} M\right) \neq 0$, there is $r_{1} \in\left(N:_{R} M\right)$ and $n_{1} \in N$ with $r_{1} n_{1} \neq 0$. Now $0 \neq\left(a+r_{1}\right)\left(m+n_{1}\right) \in N$ with $m+n_{1} \in M \backslash N$ shows that $a+r_{1}$ is nwp to $N$. Thus $a+r_{1} \in P$ with $r_{1} \in P$ implies that $a \in P$.

Therefore $S(N) \subseteq P$ and so $S(N)=P$ which implies that $N$ is $P$-primal.

Let $R$ be a commutative ring and let $M$ be an $R$-module. We recall from [3] that a submodule $N$ of $M$ is said to be a weakly prime submodule if whenever $0 \neq \mathrm{rm} \in N$, for some $r \in R$ and $m \in M$, then either $m \in N$ or $r M \subseteq N$. In [4] it is shown that every weakly prime ideal of a commutative ring $R$ is weakly primal. But a weakly prime submodule need not be weakly primal. We were unable to prove or disprove this result for weakly prime submodules. But if $N$ is a faithful submodule of $M$, we have the following Theorem.

Theorem 2.13. Let $R$ be a commutative ring and let $M$ be an $R$-module. Then, every faithful weakly prime submodule of $M$ is weakly primal.

Proof. Let $N$ be a weakly prime submodule of $M$ with $A n n_{R}(N)=0$. Set $P=$ $\left(N:_{R} M\right)$. For every $r \in P \backslash\{0\}$, there exists $m \in M \backslash N$ with $r m \in N$. If $r m \neq 0$, then $r \in w(N)$. If $r m=0$, as $\operatorname{Ann}_{R}(N)=0$, there exists $n \in N$ with $r n \neq 0$. Now $0 \neq r(m+n) \in N$ with $m+n \in M \backslash N$ implies that $r$ is nwp to $N$, that is $r \in w(N)$. Hence $P \backslash\{0\} \subseteq w(N)$. Now assume that $r \in w(N)$. There exists $m \in M \backslash N$ with $0 \neq r m \in N$. As $N$ is weakly prime we get $r \in\left(N:_{R} M\right) \backslash\{0\}$. Hence $w(N) \subseteq P \backslash\{0\}$. We have already shown that $P=w(N) \cup\{0\}$. Hence $N$ is $P$-weakly primal.

\section{Modules of fractions}

Let $R$ be a commutative ring, $S$ a multiplicatively closed subset of $R$ and $M$ an $R$ module. Consider the $S^{-1} R$-module $S^{-1} M$, the module of fractions of $M$ with respect to $S$. In this section we will discuss on the relations between the classes of weakly primal submodules of $M$ and weakly primal submodules of $S^{-1} M$. First consider the following Proposition.

Proposition 3.1. Let $R$ be a commutative ring and $S$ a multiplicatively closed subset of $R$. Let $N$ be a P-weakly primal submodule of $M$ with $P \cap S=\emptyset$. Then the following hold:

(1) If $0 \neq m / s \in S^{-1} N$, then $m \in N$.

(2) If $S$ consists of $M$-regular elements, then $S^{-1}\left(N:_{R} M\right)=\left(S^{-1} N:_{S^{-1} R} S^{-1} M\right)$.

\section{Proof.}

(1) There exists $n \in N$ and $t \in S$ with $m / s=n / t$. Hence $0 \neq u t m=u s n \in N$ for some $u \in S$. If $m \in M \backslash N$, then $u t$ is $n w p$ to $N$, that is $u t \in P \cap S$ a contradiction. 
(2) Clearly $S^{-1}\left(N:_{R} M\right) \subseteq\left(S^{-1} N:_{S^{-1} R} S^{-1} M\right)$. On the other hand, assume that $r / s \in\left(S^{-1} N: S^{-1} R S^{-1} M\right)$. For every $m \in M,(r m) / s=(r / s)(m / 1) \in S^{-1} N$. If $(\mathrm{rm}) / \mathrm{s}=0$, there exists $t \in S$ with $\mathrm{trm}=0$. Hence $r m=0 \in N$. If $(\mathrm{rm}) / \mathrm{s} \neq 0$, then $r m \in N$ by (1). This implies that $r \in\left(N:_{R} M\right)$, that is $r / s \in S^{-1}\left(N:_{R} M\right)$. Hence $\left(S^{-1} N:_{S^{-1} R} S^{-1} M\right) \subseteq S^{-1}\left(N:_{R} M\right)$.

Let $R$ be a commutative ring, $M$ an $R$-module and $S$ a multiplicatively closed set in $R$. If $K$ is a submodule of $S^{-1} M$, define $K \cap M=v^{-1}(K)=\{m \in M \mid m / 1 \in K\}$ where $v: M \rightarrow S^{-1} M$ is the natural mapping $m \mapsto m / 1$. Clearly, $K \cap M$ is a submodule of $M$.

Proposition 3.2. Let $N$ be a $P$-weakly primal submodule of an $R$-module $M$, and $S$ a multiplicatively closed subset of $R$ which consists of $M$-regular elements with $P \cap S=\emptyset$. Then:

(1) $S^{-1} N$ is a $S^{-1} P$-weakly primal submodule of the $S^{-1} R$-module $S^{-1} M$.

(2) $N=\left(S^{-1} N\right) \cap M$.

Proof. (1) Assume that $0 / 1 \neq a / s \in S^{-1} P$. By Proposition 2.5, $P$ is a weakly prime ideal of $R$. Hence $0 \neq a \in P$ by [4, Lemma 8]. Thus there exists $m \in M \backslash N$ such that $0 \neq a m \in N$. In this case $0 \neq(a / s)(m / 1) \in S^{-1} N$ and, by Proposition 3.1, $m / 1 \notin S^{-1} N$. Hence $a / s$ is $n w p$ to $S^{-1} N$, that is $a / s \in w\left(S^{-1} N\right)$. So $S^{-1} P \backslash\{0\} \subseteq$ $w\left(S^{-1} N\right)$. Now suppose that $r / s \in w\left(S^{-1} N\right)$. Then $0 \neq(r / s)(m / t) \in S^{-1} N$ for some $m / t \in S^{-1} M \backslash S^{-1} N$. Thus, by Proposition 3.1., $0 \neq r m \in N$ with $m \in M \backslash N$ shows that $r \in P \backslash\{0\}$. Hence $r / s \in S^{-1} P \backslash\{0\}$, that is $w\left(S^{-1} N\right) \subseteq S^{-1} P \backslash\{0\}$. We have already shown that $w\left(S^{-1} N\right) \cup\{o\}=S^{-1} P$. Hence $S^{-1} N$ is $S^{-1} P$-weakly primal.

(2) Clearly $N \subseteq\left(S^{-1} N\right) \cap M$. Assume that $m \in\left(S^{-1} N\right) \cap M$. If $m=0$, then $m \in N$. If $m \neq 0$, then from $0 \neq m / 1 \in S^{-1} N$ we get $m \in N$ by Proposition 3.1. Thus $\left(S^{-1} N\right) \cap M \subseteq N$.

Proposition 3.3. Let $M$ be an $R$-module and $S$ a multiplicatively closed subset of $R$ which consists of $M$-regular and $R$-regular elements. If $K$ is a $Q$-weakly primal submodule of $S^{-1} M$, then $K \cap M$ is a $(Q \cap R)$-weakly primal submodule of $M$.

Proof. Set $N=K \cap M$ and $P=Q \cap R$. It is enough to show that $w(N)=P \backslash\{0\}$. For every $a \in w(N)$, there exists $m \in M \backslash N$ such that $0 \neq a m \in N$. It follows from $0 \neq$ $(a / 1)(m / 1)=(a m / 1) \in K$ and $m / 1 \in S^{-1} M \backslash K$ that $a / 1 \in Q \backslash\{0\}$. Thus $a \in P \backslash\{o\}$, that is $w(N) \subseteq P \backslash\{0\}$. On the other hand, for every $r \in P \backslash\{0\}$, we have $0 \neq r / 1 \in Q$. So there exists $m / s \in S^{-1} M \backslash K$ with $0 \neq(r / 1)(m / s) \in K$. Then $0 \neq(r m) / 1 \in K$. Therefore $0 \neq r m \in N$ with $m \in M \backslash N$. Thus $r \in w(N)$, that is $P \backslash\{0\} \subseteq w(N)$ as required.

Theorem 3.4. Let $P$ be a weakly prime ideal of a commutative ring $R$ and let $M$ be an R-module. Assume that $S$ is a multiplicatively closed subset of $R$ which consists of $M$-regular elements with $P \cap S=\emptyset$. Then there exists a one-to-one correspondence 
between the $P$-weakly primal submodules of $M$ and the $S^{-1} P$-weakly primal submodules of of $S^{-1} M$.

Proof. This follows from Propositions 3.2 and 3.3.

\section{References}

[1] D. D. Anderson and E. Smith, Weakly prime ideals, Houston J. Math., 29(2003), 831-840.

[2] J. Dauns, Primal modules, Comm. Algebra, 25(1997), 2409-2435.

[3] S. Ebrahimi Atani and F. Farzalipour, On weakly prime submodules, Tamkang Journal of Mathematics, 38(2007), 247-252.

[4] S. Ebrahimi Atani and A. Yousefian Darani, On weakly primal ideals(I), Demonstratio Mathematica, 40(2007), 23-32.

[5] S. Ebrahimi Atani and A. Yousefian Darani, Some remarks on primal submodules, Sarajevo Journal of Mathematics, 4(2008), 181-190.

Department of Mathematics, Guilan University, P.O. Box 1914, Rasht Iran.

E-mail: ebrahimi@guilan.ac.ir

Department of Mathematics, University of Mohaghegh Ardabili, P.O. Box 179, Ardabil, Iran.

E-mail: yousefian@uma.ac.ir 